\title{
Association between Inflammatory Cytokines and Liver Functions in Rheumatoid Arthritis Patients
}

\author{
Mohamed Abdelrhman Eltahir ${ }^{1,2}$, Kawthar Abdelgaleil Mohammedsalih ${ }^{3}$, Elhaj \\ Noureldien Mohamed ${ }^{3}$, Faisal Makki Babekir ${ }^{4}$, and Amar Mohamed Ismail ${ }^{5}$ \\ 1'Department of Clinical Chemistry, Faculty of Medical Laboratory, Sudan University of Science \\ and Technology, Khartoum, Sudan \\ ${ }^{2}$ Department of Clinical Chemistry, Faculty of Medical Laboratory, Gadarif University, Khartoum, \\ Sudan \\ ${ }^{3}$ Department of Hematology, Faculty of Medical Laboratory, Sudan University of Science and \\ Technology, Khartoum, Sudan \\ ${ }^{4}$ Blood Bank and Laboratory Department, Al-Amal Hospital, Khartoum, Sudan \\ ${ }^{5}$ Department of Biochemistry and Molecular Biology, Faculty of Science and Technology, Al- \\ Neelain University, Khartoum, Sudan
}

ORCID:

Mohamed Abdelrhman Eltahir: http://orcid.org/0000-0003-0172-8362

Corresponding Author: Mohamed Abdelrhman Eltahir;

Department of clinical chemistry, faculty of medical laboratory, Sudan University of Science and Technology, Khartoum, Sudan. email: alsahaf33@yahoo.com

Received 23 April 2021

Accepted 6 June 2021

Published 30 June 2021

Production and Hosting by Knowledge E

(c) Mohamed Abdelrhman Eltahir et al. This article is distributed under the terms of the Creative Commons

Attribution License, which permits unrestricted use and redistribution provided that the original author and source are credited.

Editor-in-Chief:

Prof. Mohammad A. M. Ibnouf

\section{Abstract}

Background: Rheumatoid arthritis (RA) is associated with abnormal liver tests, and the medications used for RA are often hepatotoxic. Therefore, this study aimed to investigate an association between pro-inflammatory and anti-inflammatory cytokines and liver function tests in RA patients.

Methods: In this descriptive cross-sectional study, 88 RA patients were included, 84 of them were women and 4 men, aged 21-81 years. Serum interleukin-10 (IL-10), interleukin-17 (IL-17), Osteopontin (OPN) were measured and liver function tests were conducted.

Results: The frequency of RA was higher among adults aged $>41$ years $(72$ [81.8\%]) than young adults aged $\leq 41$ years (16 [18.2\%]). RA was more common in women (84 [95.5\%]) than in men (4 [4.5\%]) - approximately 21:1-fold. Young adults had higher abnormal IL-10 than adult RA patients (OR $=3.72, p$-value 0.044). Abnormal IL-17 (OR $=5.67$, $p$-value 0.034$)$ was found to be increased in young-adult RA patients. No association was observed between age and OPN and between the duration of disease and IL-10, IL-17, and OPN. Similarly, no association was noted between the types of treatment and IL-10, IL-17, and OPN, nor between IL-10, IL-17, OPN and liver parameters (AST, ALT, ALP, ALB, TP, and GGT).

Conclusion: Pro-inflammatory and anti-inflammatory cytokines are not associated with abnormal liver functions, as has been demonstrated in RA patients.

Keywords: rheumatoid arthritis, interleukin, liver function tests, cytokines

\section{S OPEN ACCESS}




\section{Introduction}

Rheumatoid arthritis (RA) is a common autoimmune inflammatory disease. Although the prevalence of RA is lower globally $(0.5-1 \%)$, it is associated with socioeconomic burden and higher risk of mortality rate [1]. Recent studies have demonstrated that the treatments used for RA improved outcome, and also accounts as a risk for hepatic complications [2]. The adverse effects of RA treatments include asymptomatic elevations of liver enzyme, fibrosis, and sometimes fatal hepatic necrosis [3]. On the other hand, liver disorders have been noted in untreated RA patients [4].

Increasing amounts of interleukin-10 (IL-10), a potent anti-inflammatory cytokine [5], can be detected in the synovium of RA patients. Additionally, considering that the activity of RA cannot be attenuated by IL-10 administration [6], many researchers suggest that IL-10 plays an important role in chronic liver diseases [7]. Interleukin-17 (IL-17), a proinflammatory cytokine, is upregulated in many autoimmune diseases such as RA; high levels of IL-17 have been reported to be produced in different samples of RA [8, 9]. Some investigators suggest that IL-17 plays a key role in many liver diseases and is also associated with the progress of the disease [10-12]. Osteopontin (OPN) is a proinflammatory cytokine that induces RA [13-15], and included in many liver diseases, despite its role in liver problems are still controversial [16]. Therefore, this study was carried out to find out the association between pro-inflammatory, anti-inflammatory cytokines and liver function tests among RA patients.

\section{Materials and Methods}

This descriptive cross-sectional hospital-based study was conducted on 88 RA patients who were clinically diagnosed according to the criteria of the American College of Rheumatology (ACR) 1987 and were examined at the common RA clinics in Khartoum State (military, Alamal hospital, and Zain clinic). All patients received treatment; the demographic data, type of treatment, and duration of disease for each patient were recorded -4 men and 84 women aged 28-90 years. Non-Sudanese patients with RA and those with unclear diagnosis were excluded. Serum from each subject were centrifuged at $3000 \mathrm{~g}$ for $10 \mathrm{~min}$ after clotting for $30 \mathrm{~min}$ at room temperature and stored at $-40^{\circ} \mathrm{C}$ until analysis. All samples were investigated for OPN, IL-17, and IL10 by sandwich enzyme-linked immune sorbent assay (ELISA) (ELISA Development; Thermo Fisher scientific Systems, USA) according to the manufacturer's instructions. In addition, liver functions tests (TP, Albumin, AST, ALT, GGT, and ALP) were done using 
fully automated Mindray chemistry analyzer (BS 200). Data were statistically analyzed by statistical software package, version 16. Results were expressed as numbers and percentages. Chi-square test was used to determine the level of significance $(P$-value of 0.05 was considered to be statistically significant).

\section{Results}

RA is more common in adults (72 [81.8\%]) than young adults (16 [18.2\%]), the frequency of RA was found to be higher in women (84 [95.5\%]) than in men (4 [4.5\%]). Moreover, 52 (59.1\%) patients were receiving steroids while 36 (40.9\%) were on non-steroid treatment. The duration of disease for 62 (70.5\%) patients was $\leq 6$ years and for 26 (29.5\%) was $>6$ years. Abnormal IL-10 was found in 63 (71.6\%) patients, while 25 (28.4\%) had a normal percentage. The results of characteristic data show that while 80 (91\%) RA patients had normal IL-17, 8 (9\%) had abnormal. Normal OPN was observed in 76 (86.4\%) RA patients and abnormal OPN in 12 (13.6\%) (Table 1). Chi-square analysis revealed that young adults group had a higher abnormal IL-10 than adult RA patients (OR $=3.72, p$-value 0.044). Also, abnormal IL-17 (OR $=5.67, p$-value 0.034$)$ was found to be increased in young adult RA patients while no association was seen between age and OPN (OR $=2.67$, $p$-value 0.144; Table 2). Furthermore, no association was reported between the duration of the disease and IL-10, IL-17, and OPN with p-values 0.410, 0.176, and 0.502 and OR $0.77,0.37$, and 1.30 , respectively (Table 3 ). Similarly, no association could be derived between the types of treatment and IL-10, IL-17, and OPN with $p$-value $0.246,0.286$, and 0.351 and OR 1.53, 2.21, and 0.65, respectively (Table 4). Pearson's correlation analysis revealed that there were no association between IL-10, IL-17, OPN and liver parameters (AST, ALT, ALP, ALB, TP, and GGT; Table 5).

\section{Discussion}

Abnormal liver functions were observed in RA patients. The researchers further attributed the abnormality to immune aggregations and others justified it by drugs toxicity. Accordingly, this study was carried out to assess whether the pro-inflammatory or anti-inflammatory cytokines are associated with liver functions in RA patients.

The current study revealed that there is no association between interleukins and liver function tests. In fact, abnormal liver tests were noted in patients with RA [17]. Concurrent with many previous studies, the frequency of RA is higher in elderly subjects [18, 19]. A possible explanation might be that the protective mechanisms in elderly population are 
TABLE 1: Demographic and baseline characteristics of RA Patients.

\begin{tabular}{|c|c|}
\hline Variables & Frequency (\%) \\
\hline \multicolumn{2}{|l|}{ Age (yr) } \\
\hline$\leq 41$ & 16 (18.2\%) \\
\hline$>41$ & 72 (81.8\%) \\
\hline \multicolumn{2}{|l|}{ Sex } \\
\hline Male & $4(4.5 \%)$ \\
\hline Female & 84 (95.5\%) \\
\hline \multicolumn{2}{|l|}{ Treatment } \\
\hline Steroid & 52 (59.1\%) \\
\hline Non-steroid & 36 (40.9\%) \\
\hline \multicolumn{2}{|l|}{ Duration (yr) } \\
\hline$\leq 6$ & 62 (70.5\%) \\
\hline$>6$ & 26 (29.5\%) \\
\hline \multicolumn{2}{|l|}{ Cut-off IL-10 } \\
\hline Abnormal & 63 (71.6\%) \\
\hline Normal & 25 (28.4\%) \\
\hline \multicolumn{2}{|l|}{ Cut-off IL-17 } \\
\hline Abnormal & $8(9 \%)$ \\
\hline Normal & 80 (91\%) \\
\hline \multicolumn{2}{|l|}{ Cut-off OPN } \\
\hline Abnormal & 12 (13.6\%) \\
\hline Normal & 76 (86.4\%) \\
\hline Total & 88 (100\%) \\
\hline
\end{tabular}

TABLE 2: Association between interleukins IL10, IL17, OPN and age groups.

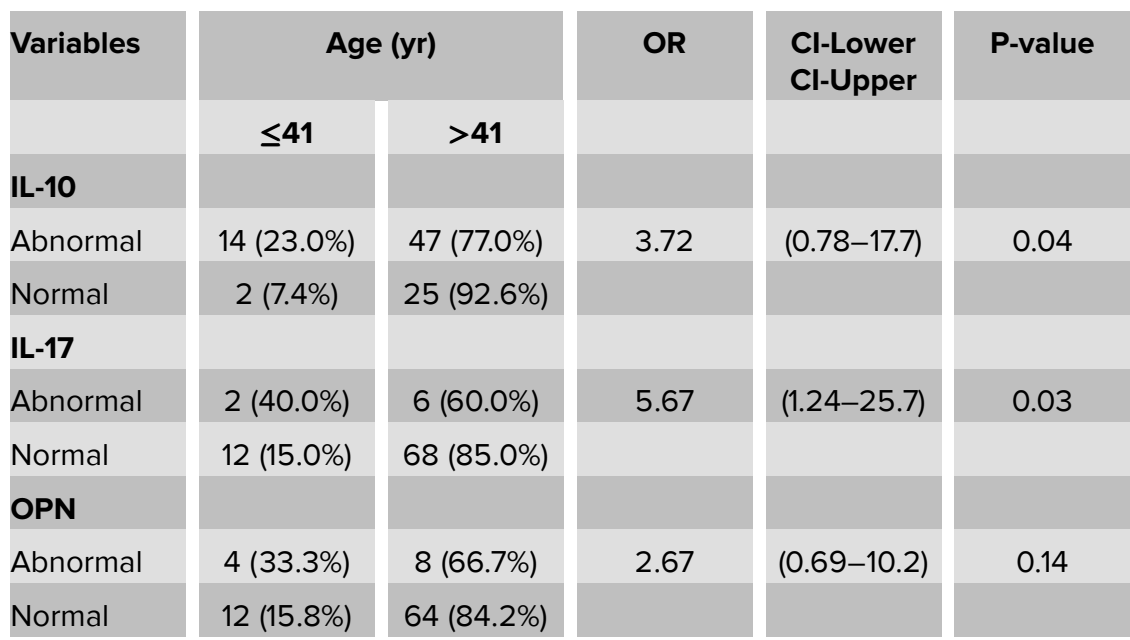

decreased, resulting in decreased immunotolerance and decreased cytokines synthesis and T cells proliferation [20]. The demographic data indicated that the prevalence of RA was found to be 21 -fold higher in women than in men. In contrast to a previous study in Sudan, the female-to-male ratio was 9:1 [21]. Since the change in sex hormones after 
TABLE 3: Association between interleukins IL10, IL17, OPN and duration of RA.

\begin{tabular}{|c|c|c|c|c|c|}
\hline \multirow[t]{2}{*}{ Variables } & \multicolumn{2}{|c|}{ Duration (yr) } & \multirow[t]{2}{*}{ OR } & \multirow{2}{*}{$\begin{array}{l}\text { Cl-Lower } \\
\mathrm{Cl} \text {-Upper }\end{array}$} & \multirow[t]{2}{*}{ P-value } \\
\hline & $\leq 6$ & $>6$ & & & \\
\hline \multicolumn{6}{|l|}{ IL-10 } \\
\hline Abnormal & 42 (68.9\%) & 19 (31.1\%) & 0.77 & (0.28-2.13) & 0.41 \\
\hline Normal & 20 (74.1\%) & 7 (25.9\%) & & & \\
\hline \multicolumn{6}{|l|}{ IL-17 } \\
\hline Abnormal & 4 (50.0\%) & 4 (50.0\%) & 0.37 & (0.08-1.65) & 0.17 \\
\hline Normal & 58 (72.5\%) & 22 (27.5\%) & & & \\
\hline \multicolumn{6}{|l|}{ OPN } \\
\hline Abnormal & 9 (75.0\%) & 3 (25.0\%) & 1.30 & $(0.32-5.25)$ & 0.50 \\
\hline Normal & 53 (69.7\%) & $23(30.3 \%)$ & & & \\
\hline
\end{tabular}

TABLE 4: Association between interleukins IL10, IL17, OPN and types of treatment.

\begin{tabular}{|c|c|c|c|c|c|}
\hline \multirow[t]{2}{*}{ Variables } & \multicolumn{2}{|c|}{ Treatment } & \multirow[t]{2}{*}{ OR } & \multirow{2}{*}{$\begin{array}{l}\text { Cl-Lower } \\
\text { Cl-Upper }\end{array}$} & \multirow[t]{2}{*}{ P-value } \\
\hline & Steroid & Non-steroid & & & \\
\hline \multicolumn{6}{|l|}{ IL-10 } \\
\hline Abnormal & 38 (62.3\%) & 23 (37.7\%) & 1.53 & $(0.61-3.83)$ & 0.24 \\
\hline Normal & 14 (51.9\%) & 13 (48.1\%) & & & \\
\hline \multicolumn{6}{|l|}{ IL-17 } \\
\hline Abnormal & 6 (75.0\%) & 2 (25.0\%) & 2.21 & $(0.42-11.6)$ & 0.28 \\
\hline Normal & 46 (57.5\%) & 34 (42.5\%) & & & \\
\hline \multicolumn{6}{|l|}{ OPN } \\
\hline Abnormal & 6 (50.0\%) & 6 (50.0\%) & 0.65 & (0.19-2.21) & 0.35 \\
\hline Normal & 46 (60.5\%) & 30 (39.5\%) & & & \\
\hline
\end{tabular}

puberty is associated with high prevalence of RA in women, a woman's immune system is potentially more reactive than that of a man. The current study reports that young adults are more likely to have abnormal IL-10 and IL-17. However, these results disagree with previous studies $[22,23]$. No association was found between age and OPN level. Concurrent with this finding, a relationship between age and OPN has been previously reported [24]. Similar to other results, no associations between IL10, IL17, OPN levels and the duration of disease have been demonstrated $[22,23,25]$. Despite reducing IL-17 after the use of steroids therapy, IL-10 was increased [26]. The present study revealed no associations between IL-10, IL-17, OPN levels and the types of treatment. It has become clear that steroids directly modulate the pro-inflammatory cytokine or suppress cytokines-producing cells [26, 27]. 
TABLE 5: Association between cytokines and liver function parameters (Pearson's correlation results).

\begin{tabular}{|c|c|c|c|}
\hline \multicolumn{2}{|c|}{ Parameters } & \multirow{2}{*}{$\begin{array}{c}\text { P-value } \\
0.62\end{array}$} & \multirow{2}{*}{$\begin{array}{c}\mathbf{R}^{2} \\
0.12\end{array}$} \\
\hline IL-10 & AST & & \\
\hline & ALT & 0.20 & 0.66 \\
\hline & ALP & 0.80 & 0.05 \\
\hline & ALB & 0.16 & -0.13 \\
\hline & TP & 0.56 & 0.02 \\
\hline & GGT & 0.25 & 0.15 \\
\hline \multirow[t]{6}{*}{ IL-17 } & AST & 0.18 & -0.15 \\
\hline & ALT & 0.82 & 0.02 \\
\hline & ALP & 0.82 & -0.02 \\
\hline & ALB & 0.23 & 0.12 \\
\hline & TP & 0.59 & 0.05 \\
\hline & GGT & 0.17 & -0.14 \\
\hline \multirow[t]{6}{*}{ OPN } & AST & 0.50 & 0.07 \\
\hline & ALT & 0.25 & 0.12 \\
\hline & ALP & 0.89 & 0.01 \\
\hline & ALB & 0.29 & -0.11 \\
\hline & TP & 0.49 & 0.07 \\
\hline & GGT & 0.98 & -0.02 \\
\hline
\end{tabular}

\section{Conclusion}

The data of present study shows that women are at a higher risk to have RA. Moreover, young adult RA patients are more likely to have abnormal IL-10 and IL-17. Furthermore, pro-inflammatory and anti-inflammatory cytokines are not associated with abnormal liver functions as has been demonstrated in RA patients.

\section{Acknowledgements}

The authors would like to express their sincere gratitude to the Dr. Mariam Abbas for her consultation and wisdom. May God bless her.

\section{Ethical Considerations}

Ethical permits for the study were obtained from the ethical review committees at the sites where patients were recruited, and all patients gave informed consent for their participation in the study. 


\section{Competing Interests}

The authors declare no known conflicts of interest in relation to this paper.

\section{Availability of Data and Material}

The study data are available with the author upon reasonable request

\section{Funding}

None.

\section{References}

[1] Kwan Ho, T. C., Mok, C. C., Cheung, T. T., et al. (2019). Management of rheumatoid arthritis. Clinical Rheumatology, vol. 38, pp. 3331-3350.

[2] Sundbaum, J. K., Eriksson, N., Hallberg, P., et al. (2019). Methotrexate treatment in rheumatoid arthritis and elevated liver enzymes: a long-term follow-up of predictors, surveillance, and outcome in clinical practice. International Journal of Rheumatic Disease, vol. 22, no. 7, pp. 1226-1232.

[3] Conway, R. and Carey, J. (2017). Risk of liver disease in methotrexate treated patients. World Journal of Hepatology, vol. 9, no. 26, pp. 1092-1100.

[4] Rakuomi, O., Go, O., Kamau, E., et al. (2017). Prevalence of abnormal liver function tests in rheumatoid arthritis. African Journal of Rheumatology, vol. 5, no. 1, pp. 70-75.

[5] Shikhpour, E., Noorbakhsh, P., Elnaz, F., et al. (2018). A survey on the role of interleukin 10 in breast cancer. Report of Biochemistry and Molecular Biology, vol. 7, no. 1, pp. $1-10$.

[6] Holdsworth, S. R. and Yi, G. P. (2015). Cytokines: names and numbers you should care about. Clinical Journal of the American Society of Nephrology, vol. 10, no. 12, pp. 2243-2254.

[7] Zhang, L. J. and Wang, X. Z. (2006). Interleukin-10 and chronic liver disease. World Journal of Gastroenterology, vol. 12, no. 11, pp. 1681-1685.

[8] Elvira, D., Nasrul, E., Sofyan, Y., et al. (2018). Increased serum levels of interleukin17 and transforming growth factor in patients with Graves' disease. Earth and Environmental Science, vol. 125, pp. 1-4. 
[9] Mengesha, B. G. and Conti, H. R. (2017). The role of IL-17 in protection against mucosal candida infections. Journal of Fungi, vol. 3, no. 52, pp. 1-12.

[10] Du, W. J., Zhen, J. H., Zeng, Z. Q., et al. (2013). Expressing of Interleukin-17 associated with disease progression and liver fibrosis with hepatitis B virus infection: IL-17 in HBV infection. Diagnostic Pathology, vol. 8, pp. 1-7.

[11] Tan, Z., Qian, X., Jiang, R., et al. (2013). IL-17A play acritical role in the pathogenesis of liver fibrosis through hepatic stellate cell activation. Journal of Immunology, vol. 191, no. 4, pp. 1-11.

[12] Zheng, L., Chu, J., Shi, Y., et al. (2013). Bone marrow-derived stem cells ameliorate hepatic fibrosis by down-regulating interleukin-17. Cell \& Bioscience, vol. 3, article 46.

[13] Shi, L., Shi, L., Wang, X., et al. (2018). Regulatory Roles of osteopontin in production of monocyte-origin MCP-1. Cell Transplantation, vol. 27, no. 8, pp. 1185-1194.

[14] Athanasiadous, D., Jiang, W., Goldbaum, D., Saleem, A., et al. (2018). Nanostructure, osteopontin, and mechanical properties of calcitic avian eggshell. Science Advances, vol. 4, no. 3219, pp. 1-13.

[15] Luukkonen, J., Pascual, M. L., Patlaka, C., et al. (2017). Increased amount of phosphorylated proinflammatory osteopontin in rheumatoid arthritis synovia is associated to decreased tartrate-resistant acid phosphatase 5B/5A ratio. PLOS ONE, vol. 12, no. 8, pp. 1-15.

[16] lida, T., Wagatsuma, K., Hirayama, D., and et al. (2018). Is osteopontin a friend or foe of cell apoptosis in inflammatory gastrointestinal and liver diseases? International Journal of Molecular Sciences, vol. 19, no. 7, pp. 1-15.

[17] Dinić, R. B., Rajković, T. S., Zivkovic, V., et al. (2018). Clinical connection between rheumatoid arthritis and liver damage. Rheumatology International, vol. 38, no. 5, pp. 715-724.

[18] Mursal, T., Elbager, S., Fadl Elmola, A., et al. (2016). Differential diagnosis of anemia in rheumatoid arthritis Sudanese patients. World Journal of Pharmaceutical and Medical Research, vol. 2, no. 4, pp. 1-4.

[19] Elsedig, M. I., Elhag, W., Elmak, M. J., et al. (2014). Seroprevalence of human parvovirus $\mathrm{B}_{19}$ antibody among Sudanese patients with rheumatoid arthritis. American Journal of Ethnomedicine, vol. 1, pp. 402-407.

[20] Kobak, S. and Bes, C. (2018). Autumn late: geriatric rheumatoid arthritis. Therapeutic Advances in Musculoskeletal Disease, vol. 10, no. 1, pp. 3-11. 
[21] Abdelsalam, S. K., Hashim, N. T., Elsalamabi, E. M., et al. (2011). Periodontal status of rheumatoid arthritis patients in Khartoum state. BMC Research Notes, vol. 4, article 460.

[22] Abd Elazeem, I. M., Mohammed, A. R., and Abdallah, H. N. (2018). Correlation of serum interleukin-10 with disease activity and severity in systemic lupus erythematosus. Egyptian Rheumatology and Rehabilitation, vol. 45, no. 1, pp. 1-9.

[23] Akdeniz, S., Akdeniz, O., Sakalli, N., et al. (2018). The relationship between interleukin-17 and osteoporosis in patients with rheumatoid arthritis. Turkish Journal of Osteoporosis, vol. 24, pp. 46-52.

[24] Iwadate, H., Kobayashi, H., Kanno, T., et al. (2013). Plasma osteopontin is correlation with bone resorption marker in rheumatoid arthritis patients. International Journal of Rheumatic Disease, vol. 17, no. 1, pp. 50-56.

[25] Al Zifzaf, S. D., Mokbel, N. A., and Abdelaziz, M. D. (2015). Interleukin-17 in Bechet's disease: relation with clinical picture and disease activity. Egyptian Rheumatology and Rehabilitation, vol. 42, no. 2, pp. 34-38.

[26] Negera, E., Walker, S., Bobosha, K., et al. (2018). The effects of prednisolone treatment on cytokine expression in patients with Erythema Nodosum Leprosum reactions. Frontiers in Immunology, vol. 9, pp. 1-15.

[27] Noack, M., Ndongo, N., and Miossec, P. (2016). Evaluation of anti-inflammatory effects of steroids and arthritis-related biotherapies in an in vitro coculture model with immune cells and synoviocytes. Frontiers in Immunology, vol. 7, pp. 1-10. 